\title{
Dynamics of Structural Transformations between Lamellar and Inverse Bicontinuous Cubic Lyotropic Phases
}

\author{
Charlotte E. Conn, ${ }^{1}$ Oscar Ces, ${ }^{1}$ Xavier Mulet, ${ }^{1}$ Stephanie Finet, ${ }^{2}$ Roland Winter, ${ }^{3}$ \\ John M. Seddon, ${ }^{1}$ and Richard H. Templer ${ }^{1, *}$ \\ ${ }^{1}$ Department of Chemistry, Imperial College London, Exhibition Road, London, SW7 2AZ, United Kingdom \\ ${ }^{2}$ European Synchrotron Radiation Facility, Boîte Postale 220, F-38043 Grenoble Cedex, France \\ ${ }^{3}$ Department of Chemistry, Physical Chemistry I, University of Dortmund, Otto-Hahn-Straße 6, D-44227 Dortmund, Germany
}

(Received 21 October 2005; published 16 March 2006)

The liquid crystalline lamellar $\left(L_{\alpha}\right)$ to double-diamond inverse bicontinuous cubic $\left(Q_{\mathrm{II}}^{D}\right)$ phase transition for the amphiphile monoelaidin in excess water exhibits a remarkable sequence of structural transformations for pressure or temperature jumps. Our data imply that the transition dynamics depends on a coupling between changes in molecular shape and the geometrical and topological constraints of domain size. We propose a qualitative model for this coupling based on theories of membrane fusion via stalks and existing knowledge of the structure and energetics of bicontinuous cubic phases.

Lyotropic liquid crystalline phases form when amphiphilic molecules are mixed with a polar solvent $[1,2]$. The many structures they can adopt, their complex phase behavior and their energetics have received a great deal of attention over the past half century. Much is now understood about their equilibrium behavior and this has informed the development of surfactant technologies and our understanding of how amphiphiles in biological membranes can control biochemical activity [3,4]. By comparison the study of the nonequilibrium structure and dynamics of lyotropic liquid crystalline systems is in its infancy and little is understood about the mechanisms by which one phase transforms into another [5,6]. Understanding the dynamics of lyotropic phase transformations is of practical interest for applications where amphiphilic materials are processed and of relevance in biological systems where membrane fusion or division occurs.

The present work focuses on a phase transition where membrane fusion occurs: the fluid lamellar to inverse bicontinuous cubic phase transition, Fig. 1. Models for the process of membrane fusion between apposed lipid bilayers have been proposed by a number of groups [79]. All rely on the formation of transient lipid contacts known as stalks, which subsequently break through to form the beginnings of the tubular connections that are the fundamental connecting element in the inverse bicontinuous cubic phases. Recent theoretical models of the structure and energy of the stalk have incorporated both elastic energy, accounting for the splay, saddle splay, and tilt deformations of the membrane, as well as hydration repulsion acting between the apposing membranes [10-12]. The models have been used to estimate the energy of stalk formation and mesoscopic models have been used to simulate the entire fusion process [13]. However, very few measurements have been made to test these models. To date the most convincing evidence has been structural evidence that is consistent with the existence of fusion stalks [14]. As for the structural dynamics, a limited amount of experimental work has been carried out on the lamellar to inverse bicontinuous cubic phase transition [15-19]. The major problem to date with these dynamical studies is the lack of reproducibility in results $[20,21]$.

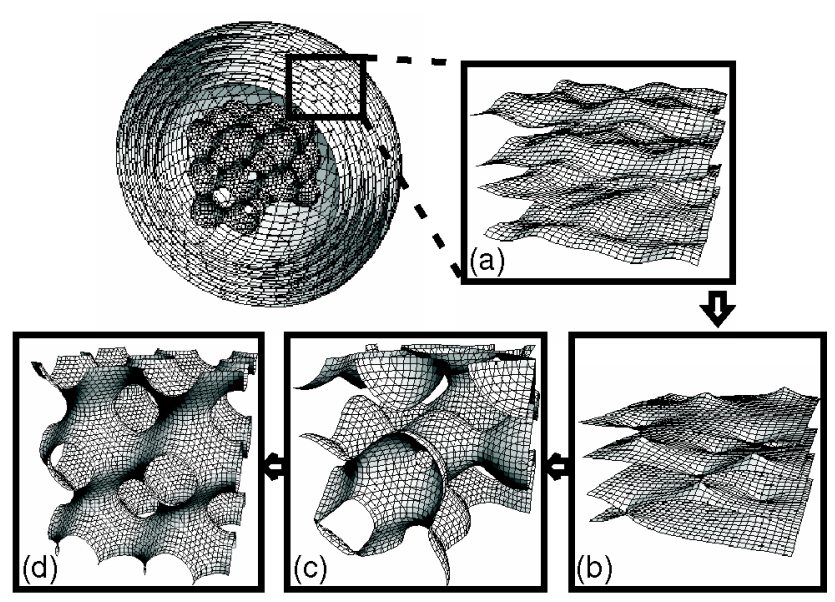

FIG. 1 (color online). In these images we show the structural transformations observed in our experiments by sketching the bilayer midplane. The figures are scaled relative to each other to reflect our experimental observations. A spherical onion vesicle consisting of concentric bilayers is shown including the swollen cubic phase present at the center. Thermal undulations in the lamellar phase (a) disappear as interlamellar attachments (ILAs) are formed (b). This occurs because the bilayer's area and the volume it circumscribes are fixed in an onion vesicle and hence forming contacts exerts a lateral tension. When the tension reaches a critical value, ILAs rupture and form a disordered network of funnels (c) through which water flows from the center of the vesicle. The disordered sponge structure soon resolves into ordered bicontinuous cubic structures such as the swollen $P$ cubic (d). 
We have overcome the variability in such dynamical measurements and this has allowed us for the first time to record the dynamical process reproducibly in temperature and pressure-jump experiments. In this Letter we present a systematic body of experimental data on the fluid lamellar to inverse bicontinuous cubic phase transition in the binary system of the monoacylglyceride, monoelaidin (ME) in excess water and suggest a qualitative hypothesis for the sequence of events that we observe.

Lyophilised ME (Larodan Fine Chemicals, Sweden) was mixed with at least $80 \mathrm{wt} \%$ triply distilled and deionised water, much larger than the limiting excess water hydration of $70 \mathrm{wt} \%$. To make our kinetic measurements reproducible the sample was subjected to 30 thermal cycles between -20 and $70^{\circ} \mathrm{C}$. We have previously observed such cycling to lead to a gradual reduction in average domain size, as evidenced by the reduced granularity of the powder diffraction pattern. In total more than 100 pressure and temperature jumps were analyzed, all of which displayed good reproducibility and clear trends in behavior with changes in thermodynamic parameters.

All transitions were monitored using time-resolved small angle x-ray scattering. Pressure-jump experiments were carried out at the ID02 beam line of the European Synchrotron Radiation Facility in Grenoble, France using a high pressure $\mathrm{x}$-ray sample cell capable of altering hydrostatic pressure within $7 \mathrm{~ms}$ [22]. Temperature-jump experiments were made using a custom-designed kinetics beam line at Imperial College London with a measured x-ray flux

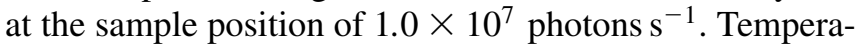
ture jumps, using thermoelectric heating, were made at a rate of $50{ }^{\circ} \mathrm{C} \mathrm{min}^{-1}$ as measured in the sample cell [23].

The dynamics of the $L_{\alpha}$ to $Q_{\mathrm{II}}^{D}$ phase transition, brought about by rapid changes in temperature or pressure, have a number of common features, as shown in Fig. 2. Most importantly, we find the dynamics are only reproducible if samples are composed of topologically distinct, physically separated domains with a narrow size distribution (see Cryo-TEM section below, $\approx 200 \pm 20 \mathrm{~nm}$ diameter in our case). This reproducibility can be clearly seen in

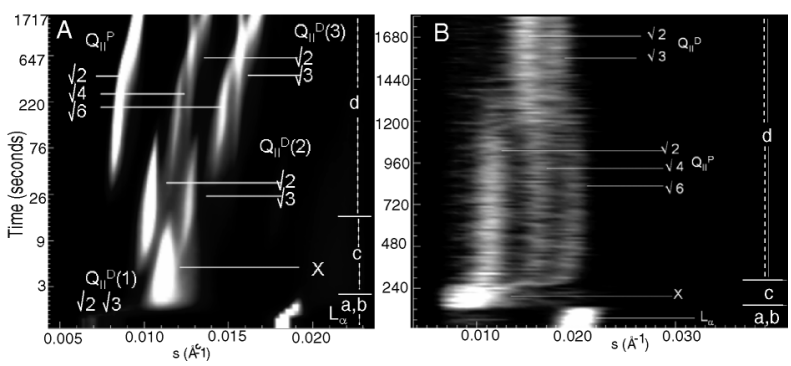

FIG. 2. A pressure jump from 1100 to $260 \mathrm{bar}, T=46.7^{\circ} \mathrm{C}$ (a) and a temperature jump from $30^{\circ} \mathrm{C}$ to $60^{\circ} \mathrm{C}, p=1$ bar (b) are shown. Each 2D image is integrated to produce a 1D plot of intensity versus scattering vector, $s$, which is then stacked as a function of time. Regions $\mathrm{a}, \mathrm{b}, \mathrm{c}$, and $\mathrm{d}$ correspond to the structural intermediates depicted in Fig. 1.
Fig. 3 which plots the lattice parameter as a function of time for the pressure and temperature jumps shown in Fig. 2. A second set of data obtained from a jump of the same amplitude has been added to each plot. With regards to the characteristic time scales the two plots are virtually indistinguishable. Some small, systematic differences can occasionally be seen in the plots of lattice parameter as a function of time; these are, however, of the same order as the noise in the signal. Reproducibility was checked for every sample.

We always observe a weak and previously unreported signal from a highly swollen bicontinuous cubic phase, $Q_{\mathrm{II}}^{D}$, of maximum spacing $232 \AA$, in coexistence with the fluid lamellar phase. The lamellar gel phase, which occurs below $15^{\circ} \mathrm{C}$ at atmospheric pressure and above 1300 bar at $46.7^{\circ} \mathrm{C}$, also coexists with a swollen primitive $Q_{\mathrm{II}}^{P}$ cubic phase of maximum lattice parameter $311 \AA$. Only two peaks were detectable for these phases, but the intensity ratio and indexing were consistent with previous observations. The cubic structures appear to be very stable, only being removed on freezing the chains at very high pressures or low temperatures. Following the step change in
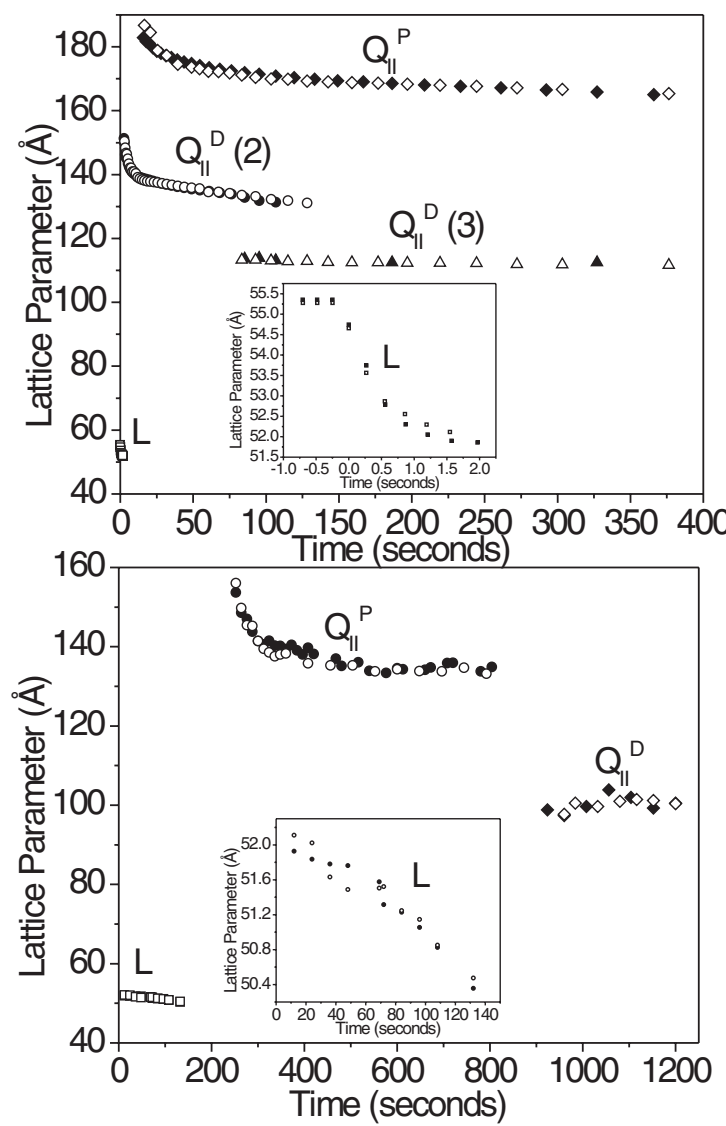

FIG. 3. The change in lattice parameter with time is shown following the pressure jump shown in Fig. 2(a) and the temperature-jump shown in Fig. 2(b). Two experiments having the same jump amplitude are plotted for each. In each case the inset is a close-up view of the behavior of the lamellar phase immediately following the jump. 
thermodynamic conditions (pressure jump or temperature jump) the lamellar phase always shrinks to a specific threshold interlamellar spacing, constant to within the resolution of our experiments before disappearing. This threshold value is remarkably constant for temperaturejump experiments even across a range of samples - over 80 temperature jumps were carried out on four different samples giving rise to an average final lattice parameter reached of $51.63 \AA$ with a standard deviation of $0.46 \AA$. The rate of decrease in interlamellar spacing increases in proportion to the temperature or pressure amplitude of the jump. During this phase the highly swollen bicontinuous cubic signal smears out, moves to higher scattering angle, and, coincident with the disappearance of the lamellar Bragg peaks, merges with a broad, featureless ring of scatter (marked $X$ in Fig. 2). After a period of a few seconds the diffuse scatter resolves itself into a set of peaks from intermediate inverse bicontinuous cubic phases, $Q_{\mathrm{II}}^{D}$ and $Q_{\mathrm{II}}^{P}$, which replace it. These intermediate cubic phases are initially significantly more hydrated than expected from equilibrium results and dehydrate over time while simultaneously their phase composition increases, Fig. 3. It seems that the mechanistic route adopted following a pressure jump is quite different to that following a $T$ jump. Thus the temperature jump seems to follow the equilibrium phase diagram, but very slowly, whereas the pressure jump induces a more complex pathway between the $L_{\alpha}$ phase and the final $Q_{\mathrm{II}}^{D}$ phase. The intermediate $Q_{\mathrm{II}}^{D}$ and $Q_{\mathrm{II}}^{P}$ phases may be used for transport of water; the small curvature elastic energy associated with expanding channel size means this can be an energetically favorable way of increasing water flux through the sample.

These dynamical features are not exclusive to ME; both the decrease in lattice parameter of the lamellar phase and the existence of an intermediate swollen cubic phase have been previously noted for several other amphiphiles including monomyristolein (unpublished data), 1-monoolein (MO) [24], and 2:1 Lauric Acid (LA) and Di-laurylphosphatidylcholine (DLPC) (2LA-DLPC) mixtures [16]. Additionally, for the systems monomyristolein in excess water and monoelaidin and monoolein under limited water conditions (unpublished data) we again observe a highly specific final lattice parameter of the lamellar phase and a strong dependence of the rate of shrinking on pressurejump amplitude. This dependence is not observed during lamellar to nonlamellar transitions which do not involve the formation of stalks. For example, while there is some small shrinking of the lamellar phase upon jumping into the fluid isotropic phase ( $\mathrm{ME}$ in $24 \mathrm{wt} \% \mathrm{H}_{2} \mathrm{O}$ ) there is no observable dependence of the rate of shrinkage on jump amplitude (unpublished data).

The fact that dynamical reproducibility is dependent on uniform domain size is strong evidence for structural changes at the scale of the unit cell being topologically and geometrically coupled to structural constraints at the nanoscale; see Fig. 1. From polarizing microscopy we know that in the lamellar phase our samples consist of closely packed onion vesicles [25]. Using cryo-TEM, we observe that upon transformation into the $Q_{\mathrm{II}}^{D}$ cubic phase, the rounded multilamellar vesicles are transformed into cubosomes [26] of approximately the same dimensions as the onion vesicles. Smooth powder diffraction patterns obtained from these samples set an upper limit of the order of $1 \mu \mathrm{m}$ on the domain size. These three observations suggest that the phase transitions that we record all occur within the confines of the vesicles we have formed. This sets significant constraints on local composition, diffusion and topology.

The fact that the disappearance of the highly swollen cubic phase after a thermodynamic jump is structurally continuous with the appearance of diffuse scatter is strong evidence that it exists inside the onion vesicle, in coexistence with the lamellar phase. This suggests that its function is to act as a seed for the growth of the bicontinuous cubic phases out of an initially only locally ordered bicontinuous phase (the source of the diffuse scatter). We hypothesize that the swollen cubic is located in the center of the onion vesicle (Fig. 1) removing the high energetic cost of filling the core with spherical bilayers of increasingly higher degrees of mechanically unfavorable curvature. It is interesting to note in this regard that the cubic phase lattice parameter is larger than the upper limit predicted by Bruinsma [27], who calculated that thermally activated vibrational modes would disorder the structure. However, with the swollen cubic phase anchored to a vesicle wall perhaps the structure becomes stabilized. When we make a thermodynamic jump we are heading in a direction in which the bicontinuous cubic phase will shrink. At the same time the lamellar lattice parameter is shrinking, which indicates that water being expelled from the cubic phase does not pass into or through the lamellar bilayers.

The shrinkage of the lamellar lattice is occurring at the same time that stalks are formed between bilayers. From equilibrium temperature studies one expects a change in lattice parameter of the fluid lamellar phase of $-0.06 \AA^{\circ} \mathrm{C}^{-1}$ and the equivalent for pressure which is $+1.5 \AA \mathrm{kbar}^{-1}$. In every case the actual changes are significantly greater than this. We hypothesize that this is due to the formation of stalks. This will produce the observed effects only if the bilayers have excess area in the form of thermal undulations [28] and, of course, these undulations are an absolute prerequisite for stalk formation itself (Fig. 1). For fixed water volume between adjacent bilayers in an onion vesicle the formation of stalks will reduce the average bilayer spacing as it irons out undulations, while simultaneously imparting lateral tension in the bilayers. We know that rupture of the stalk to form a fusion pore is dependent on the presence of sufficient lateral tension to open up the pore [8]. Hence the hypothesis is able to rationalize the very specific and reproducible value of the threshold lamellar lattice parameter prior to the phase's complete disappearance. 
The rupture of the fluid lamellar bilayer throughout the sample requires the concomitant movement of the water stored in the central, disordered bicontinuous structure through the pores (Fig. 1). This destroys lamellar symmetry, and with pores distributed over spherical surfaces and the initially rapid flow of both lipid and water there can be no initial cubic symmetry to replace it. Hence the broad unresolved peak during this stage of the process. The appearance of an initially highly swollen cubic phase from this broad peak is explained by the now slower flow of large volumes of water, allowing the reorganization of some parts of the domain into cubic regions. Regular, cubic arrays of pores are at a lower free energy than the random arrangement in phase $X$ [29] and the energetic cost of swelling the $D$ and $P$ cubic phases is not that great [2]. At fixed lipid mass within the domain the shrinkage of these intermediate cubic phases can only occur via the growth of new unit cells, and hence an increase in topology (density of channels). This, by comparison with maintaining a swollen cubic phase, is clearly energetically costly as can be seen by the slow rate of growth of the equilibrium phase (Fig. 2).

To our knowledge this is the first time that the kinetics of a bicontinuous cubic lipid phase transformation has been made entirely reproducible, emphasizing the necessity of considering nanoscale structural constraints along with changes at the molecular level on studies of phase transition kinetics. The consistent results associated with the reduction in lattice parameter of the lamellar phase, including the highly specific lattice parameter associated with rupture and the strong correlation between the rate of shrinking and the jump amplitude, directly demonstrate the effect of stalk formation on the lipid bilayer.

We acknowledge the ESRF for provision of synchrotron radiation facilities and would like to thank Julia Kraineva for assistance with the pressure-jump apparatus. We thank the EPSRC for platform Grant No. GR/S77721, for funding C.C. and, along with the Chemical Biology Centre (Imperial College London), for funding X. M.

*Corresponding author.

Email address: r.templer@imperial.ac.uk

[1] J. M. Seddon, Biochim. Biophys. Acta 1031, 1 (1990).
[2] J. M. Seddon and R.H. Templer, Phil. Trans. R. Soc. A 344, 377 (1993).

[3] E. V. Brink-van der Laan, J. A. Killian, and B. de Kruijff, Biochim. Biophys. Acta 1666, 275 (2004).

[4] O. Ces and X. Mulet, Signal Transduction (to be published).

[5] J.M. Seddon, A. Squires, O. Ces, R.H. Templer, J. Woenckhaus, and R. Winter, in Self-Assembly, edited by B.H. Robinson (IOS Press, Amsterdam, 2003), pp. $212-221$.

[6] P. Laggner and M. Kriechbaum, Chem. Phys. Lipids 57, 121 (1991).

[7] L. V. Chernomordik and M.M. Kozlov, Annu. Rev. Biochem. 72, 175 (2003).

[8] Y. Kozlovsky, L. V. Chernomordik, and M. M. Kozlov, Biophys. J. 83, 2634 (2002).

[9] D. P. Siegel, Biophys. J. 49, 1171 (1986).

[10] D. P. Siegel and M. M. Kozlov, Biophys. J. 87, 366 (2004).

[11] Y. Kozlovsky et al., Biophys. J. 87, 2508 (2004).

[12] Y. Kozlovsky and M.M. Kozlov, Biophys. J. 82, 882 (2002).

[13] M. Muller, K. Katsov, and M. Schick, Biophys. J. 85, 1611 (2003).

[14] L. Yang and H. W. Huang, Science 297, 1877 (2002).

[15] M. Caffrey, Biochemistry 26, 6349 (1987).

[16] A. M. Squires et al., Langmuir 18, 7384 (2002).

[17] V. Cherezov et al., J. Membr. Biol. 195, 165 (2003).

[18] S. M. Gruner et al., Biochemistry 27, 2853 (1988).

[19] D. P. Siegel and J. L. Banschbach, Biochemistry 29, 5975 (1990).

[20] J. Erbes et al., Phys. Chem. Chem. Phys. 2, 151 (2000).

[21] R. Winter, Biochim. Biophys. Acta 1595, 160 (2002).

[22] J. Woenckhaus et al., Rev. Sci. Instrum. 71, 3895 (2000).

[23] Computer control of all aspects of the beam line used TVX software developed in collaboration with Professors Eric Eikenberry, Paul Scherrer Institute and Sol Gruner et al., Cornell University. Data analysis, reduction, and presentation was made using AXCESS software, based on the IDL programming environment, developed in house by Andrew Heron, Imperial College London.

[24] J. Kraineva et al., Langmuir 21, 3559 (2005).

[25] L. Ramos et al., Europhys. Lett. 66, 888 (2004).

[26] K. Larsson, J. Phys. Chem. 93, 7304 (1989).

[27] R. Bruinsma, J. Phys. II (France) 2, 425 (1992).

[28] W. Helfrich, J. Phys. (France) 46, 1263 (1985).

[29] T. Charitat and B. Fourcade, J. Phys. II (France) 7, 15 (1997). 